\title{
Comparison of the Clinical Efficacy of Proximal Femoral Nail Antirotation and Femoral Head Replace- ment on Intertrochanteric Fracture and the Influence of Tartrate Resistant Acid Phosphatase $5 \mathrm{~b}$ and Bone Alkaline Phosphatase
}

\author{
G. H. MAAND Y. L. WU ${ }^{1 *}$
}

Department of Extra osseous, Qixia City People's Hospital, Qixia, Shandong 265300, ${ }^{1}$ Department of orthopaedics, Lai Zhou City People's Hospital, No. 1718, Wuli Street, Laizhou, Shandong 261400, China

Ma et al.: Clinical Efficacy of Proximal Femoral Nail Antirotation and Femoral Head Replacement on Intertrochanteric Fracture

\begin{abstract}
To explore the clinical efficacy of proximal femoral nail antirotation and femoral head replacement in the treatment of intertrochanteric fracture of femur and to explore the effect of tartrate resistant acid phosphatase 5b and bone alkaline phosphatase. 100 patients with intertrochanteric fracture of femur from January 2017 to January 2020 in our hospital were selected and randomly divided into proximal femoral nail antirotation group $(n=50)$ and femoral head replacement group $(n=50)$. The operation time, intraoperative blood loss, postoperative weight bearing time, length of hospital stay, Harris score, tartrate resistant acid phosphatase $5 \mathrm{~b}$, bone alkaline phosphatase level and complications were compared between the two groups. Compared with femoral head replacement group, the operation time, intraoperative blood loss, postoperative weight bearing time and hospitalization time of proximal femoral nail antirotation group were significantly increased $(\mathbf{p}<\mathbf{0 . 0 5})$. Compared with before operation, Harris score of two groups were significantly increased after operation and the increase of femoral head replacement group was significantly greater than that of proximal femoral nail antirotation group $(\mathbf{p}<0.05)$. Compared with before operation, tartrate resistant acid phosphatase $5 \mathrm{~b}$ was significantly lower, bone alkaline phosphatase was significantly higher and femoral head replacement was significantly higher in the two groups after operation $(\mathbf{p}<0.05)$. Compared with femoral head replacement group $(\mathbf{1 6 . 0 0} \%)$, the incidence of complications in proximal femoral nail antirotation group $(6.00 \%)$ was significantly lower $(p<0.05)$. Compared with proximal femoral nail antirotation, femoral head replacement in the treatment of intertrochanteric fracture of femur has shorter postoperative weight bearing time and hospitalization time, which is more conducive to the recovery of hip joint function and improvement of bone metabolism indexes. However, femoral head replacement has longer operation time, more bleeding and higher risk of postoperative complications, which has a good reference value for clinical treatment of intertrochanteric fracture of femur.
\end{abstract}

Key words: Proximal femoral nail antirotation, femoral head replacement, intertrochanteric fracture of femur, clinical efficacy, tartrate resistant acid phosphatase $5 \mathrm{~b}$, bone alkaline phosphatase

Intertrochanteric fracture of femur (IFF), as a common type of hip fracture, is a progressive disease caused by increased pressure in the osteofascial compartment, which often occurs in the elderly ${ }^{[1]}$. IFF can cause swelling, dyskinesia, pain and other clinical manifestations and with the aging of society, the incidence of IFF is increasing year by year, seriously threatening the quality of life of patients ${ }^{[2]}$.
Conservative treatment and surgical treatment are commonly used in clinical treatment of IFF. With the continuous clinical practice, related studies have shown that the effect of surgical treatment is more significant, which can not only quickly reduce body pain, but also shorten bed rest time ${ }^{[3]}$. At present, the commonly used surgical methods are proximal femoral nail antirotation (PFNA) and femoral head replacement (FHR). Some

*Address for correspondence

E-mail: drwuwu@126.com 
scholars believe that PFNA not only has the advantages of less bleeding and short operation time, but also has a significant long term effect ${ }^{[4,5]}$, but some studies have shown that PFNA is not conducive to the recovery of hip joint function, while FHR has a better short term effect. At present, there is still some controversy about which kind of operation should be adopted in clinic for better curative effect. Tartrate resistant acid phosphatase $5 \mathrm{~b}$ (TRACP-5b) and bone alkaline phosphatase (BALP) are commonly used markers of bone metabolism in clinic, which are closely related to the occurrence and development of IFF. However, there are few studies to explore the effects of PFNA and FHR on bone metabolic indexes in patients with IFF, which is worthy of further study. Therefore, the main purpose of this study is to explore the clinical efficacy of PFNA and FHR on IFF and the effects on TRACP-5b and BALP, in order to provide more sufficient scientific basis for clinical treatment of $\operatorname{IFF}^{[6-8]}$.

\section{MATERIALS AND METHODS}

\section{General Information:}

One hundred patients with IFF who were treated in our hospital from January 2017 to January 2020 were randomly divided into PFNA group $(n=50)$ and FHR group $(\mathrm{n}=50)$. In PFNA group, there were 19 males and 31 females and the average age was $(61.25 \pm 3.20)$ y old. The fracture side was left in 19 cases, the right side in 31 cases. The Arbeitsgemeinschaft fur Osteosynthesefragen (AO) classification of fracture was A1.1 in 18 cases, A1.2 in 12 cases, A1.3 in 13 cases and A2.1 in 7 cases. The causes of fracture were patients fall from height in 3 cases, fall in 27 cases and traffic accident in 20 cases. In FHR group, there were 18 males and 32 females, aged from 31 to $77 \mathrm{y}$, with an average age of $60.74 \pm 3.11 \mathrm{y}$. The fracture side was left in 21 cases and right in 29 cases. According to AO classification, there were 19 cases of A1.1 type, 13 cases of A1.2 type, 11 cases of A1.3 type and 7 cases of A2.1 type. The causes of fracture included fall from height in 4 cases, fall in 26 cases and traffic accident in 20 cases. There was no significant difference in general data between the two groups ( $p>0.05)$.

\section{Inclusion and exclusion criteria:}

Inclusion criteria-Patients who were diagnosed as IFF by hip computerized tomography (CT); patients with unilateral fracture; patients without fracture of other parts; patients with no obvious history of trauma at operation site; patients or their family members signed informed consent form.

Exclusion criteria-Patients with contraindications of PFNA and FHR; patients with congenital hip deformity; patients with ipsilateral osteoarthritis and rheumatoid arthritis; patients with cardiovascular and cerebrovascular diseases, kidney disease, diabetes or taking bone metabolic drugs in nearly $1 \mathrm{y}$; patients with pathological fracture caused by bone tumor and bone tuberculosis.

\section{Methods:}

\section{PFNA group:}

First, the patient was supine on the operating bed and epidural anesthesia was performed on the patient. After that, the affected limb was fixed on the traction frame, adduction and rotation were about $15^{\circ}$ and closed reduction was carried out through the traction bed under the monitoring of the $\mathrm{C}$-arm and the continuity of the inner and outer cortex of the femoral calcar was restored as much as possible. A longitudinal incision of about $5 \mathrm{~cm}$ was made at the top of the greater trochanter of the femur, stripping it layer by layer. Furthermore, the fracture site is fully exposed, the medial side of the apex of the greater trochanter is opened with the aid of the mouthpiece and the guide pin is inserted. After the C-arm confirms that the guide pin is inserted at the appropriate position, conventional reaming is carried out and then PFNA main nail with appropriate thickness and length is implanted to ensure that the main nail is in the center of the femur in the anterior and lateral positions, thus ensuring the good reduction of the fracture. Insert the guide needle into the femoral neck, the positive position is lower than the midline of the femoral head neck and the lateral position is in the middle of the femoral neck, continue reaming, implant a spiral blade of appropriate length, lock it, put the distal screw into it through the sight and screw on the tail cap. The operation area was washed repeatedly, drainage tube was indwelled at the same time and finally the incision was sutured ${ }^{[9]}$.

\section{FHR group:}

Take the patient's lateral position, under epidural anesthesia. After the anesthesia works, that is, the greater trochanter is taken as the center to make an arc shaped incision with a length of about $12 \mathrm{~cm}$. Cut the skin tissue, then it is stripped layer by layer. After the fracture is fully exposed, the joint capsule is cut open, 
the fracture part with large and small trochanter is fixed with steel wire. Osteotomy is performed $2 \mathrm{~cm}$ above the small trochanter, the femoral head is taken out, the large and small trochanter is reduced, the larger bone fragment is kept as much as possible in the process and finally fixed with Kirschner wire tension band. At the end of reaming, the artificial femoral head with lengthened or common cement handle is used to start joint replacement. After ensuring good stability of bone and joint, the operation area is repeatedly washed, drainage tube is indwelled and incision is sutured.

After operation, both groups were given routine analgesia, anticoagulation, anti-infection and other treatments and patients were encouraged to carry out knee joint and hip joint activities on the affected side and functional exercise of quadriceps femoris. X-ray reexamination was carried out after operation to ensure good fixation of the affected limb (fig. 1 and fig. 2).

\section{Observation index:}

Operative indexes-The operation time, intraoperative blood loss, postoperative weight bearing time and hospitalization time of the two groups were compared.

Hip joint function: Harris rating scale ${ }^{[10]}$ was used to score the hip joint of the two groups of patients before and 3 mo after operation, including 7 parts, with a full

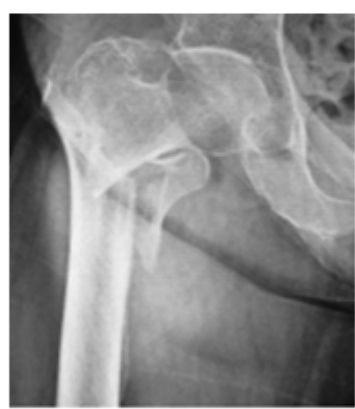

Preoperation

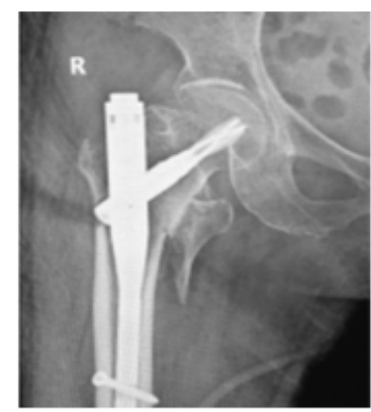

Postoperation

Fig. 1: Before and after PFNA operation

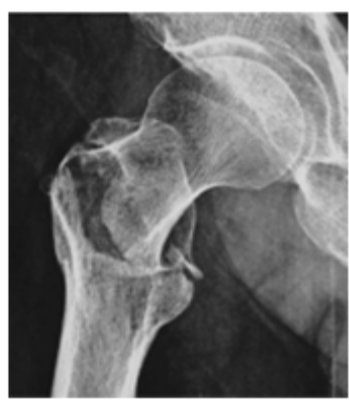

Preoperation

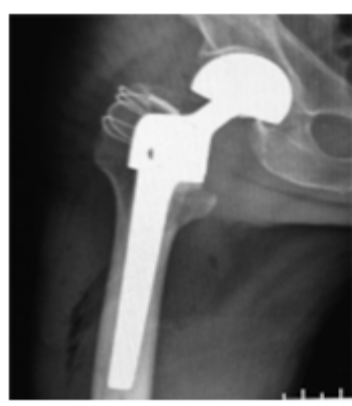

Postoperation

Fig. 2: Before and after FHR operation

score of 100 points, the higher the score, the better the recovery of the patients.

Bone metabolism index-Two groups of fasting elbow venous blood $3 \mathrm{ml}$ were taken before operation and in the morning 3 mo after operation and placed in Eppendorf (EP) tube. After $1 \mathrm{~h}$ at room temperature, the serum was separated by centrifugation, placed in dry test tube, stored at $-80^{\circ}$ for test and the serum TRACP-5b and BALP levels were detected by enzyme linked immunosorbent assay (ELISA). The kit used was provided by Shanghai Fusheng Industrial Co., Ltd.

Complications-The patients were followed up for $1 \mathrm{y}$ to observe the occurrence of urinary system infection, pressure sores, pulmonary infection, abnormal internal fixation, prosthesis dislocation, etc.

\section{Statistical analysis:}

SPSS 18.0 was used for statistical analysis. The measurement data were expressed as mean \pm standard deviation $(\overline{\mathrm{x}} \pm \mathrm{s})$ and $\mathrm{t}$ test was conducted. The counting data was expressed as case (n) or percentage (\%) and tested by $\chi^{2}, p<0.05$ indicates that the difference is statistically significant.

\section{RESULTS AND DISCUSSION}

Comparison of related surgical indexes between the two groups; the operation time and intraoperative blood loss in PFNA group were significantly longer than those in FHR group and the postoperative weight bearing time and hospital stay were significantly shorter than those in FHR group ( $\mathrm{p}<0.05)$, as shown in Table 1.

Comparison of Harris score between the two groups before and after operation; there was no significant difference in Harris score between the two groups before operation ( $p>0.05)$. After operation, Harris score of the two groups increased significantly and the increase in FHR group was significantly greater than that in PFNA group, with statistical significance $(p<0.05)$, as shown in Table 2.

Comparison of TRACP-5b and BALP levels between the two groups before and after operation; there was no significant difference in TRACP-5b and BALP levels between the two groups before operation $(p>0.05)$. After operation, the level of TRACP-5b decreased and the level of BLAP increased in the two groups. The change range in FHR group was significantly larger than that in PFNA group and the difference was statistically significant $(\mathrm{p}<0.05)$, as shown in Table 3. 
TABLE 1: COMPARISON OF RELATED SURGICAL INDEXES BETWEEN THE TWO GROUPS ( $\overline{\mathbf{x}} \pm \mathbf{s})$

\begin{tabular}{lcccc}
\hline Indicators & PFNA group $(\mathbf{n}=50)$ & FHR group $(\mathbf{n}=50)$ & $\mathrm{t}$ & $\mathrm{p}$ value \\
\hline Operation time $(\mathrm{min})$ & $53.89 \pm 10.71$ & $78.98 \pm 12.51$ & 7.527 & 0.002 \\
Intraoperative blood loss $(\mathrm{ml})$ & $144.91 \pm 32.69$ & $398.20 \pm 57.81$ & 9.341 & 0.000 \\
Postoperative weight bearing time (d) & $4.16 \pm 0.96$ & $19.27 \pm 3.94$ & 7.592 & 0.005 \\
Hospitalization time (d) & $17.75 \pm 4.32$ & $20.38 \pm 4.98$ & 6.524 & 0.017 \\
\hline
\end{tabular}

TABLE 2: COMPARISON OF HARRIS SCORE BETWEEN THE TWO GROUPS BEFORE AND AFTER

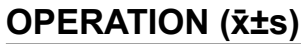

\begin{tabular}{lcccc}
\hline Time & PFNA group $(\mathrm{n}=50)$ & FHR group $(\mathrm{n}=50)$ & $\mathrm{t}$ & $\mathrm{p}$ value \\
\hline Preoperatively & $37.84 \pm 3.68$ & $37.20 \pm 3.57$ & 0.767 & 0.772 \\
Postoperatively & $71.73 \pm 4.24^{*}$ & $80.61 \pm 4.32^{*}$ & 7.014 & 0.010
\end{tabular}

Note: * indicates the comparison between the same group before and after operation, ${ }^{*} p<0.05$

TABLE 3: COMPARISON OF TRACP-5B AND BALP LEVELS BETWEEN THE TWO GROUPS BEFORE AND AFTER OPERATION ( $\bar{x} \pm s, U / L)$

\begin{tabular}{llcccc}
\hline Indicators & & PFNA group $(n=50)$ & FHR group $(n=50)$ & t & p value \\
\hline \multirow{2}{*}{ TRACP-5b } & Preoperatively & $7.94 \pm 1.82$ & $7.97 \pm 1.69$ & 0.756 & 0.779 \\
& Postoperatively & $6.67 \pm 1.08^{*}$ & $4.40 \pm 0.57^{*}$ & 5.476 & 0.031 \\
BALP & Preoperatively & $37.78 \pm 8.47$ & $37.81 \pm 8.54$ & 0.917 & 0.482 \\
& Postoperatively & $58.28 \pm 10.23^{*}$ & $65.23 \pm 13.13^{*}$ & 6.520 & 0.019 \\
\hline
\end{tabular}

Note: * indicates the comparison between the same group before and after operation, ${ }^{*} p<0.05$

TABLE 4: COMPARISON OF COMPLICATIONS BETWEEN THE TWO GROUPS [n (\%)]

\begin{tabular}{lcccc}
\hline Adverse reactions & PFNA group $(\mathrm{n}=50)$ & FHR group $(\mathrm{n}=50)$ & $\mathbf{X}^{2}$ & $\mathrm{P}$ value \\
\hline Urinary system infection & $1(2.00)$ & $1(2.00)$ & - & - \\
Pressure ulcer & $1(2.00)$ & $0(0.00)$ & - & - \\
Pulmonary infection & $1(2.00)$ & $3(6.00)$ & - & - \\
Abnormal internal fixation & $0(0.00)$ & $3(6.00)$ & - & - \\
Dislocation of prosthesis & $0(0.00)$ & $1(2.00)$ & - & - \\
Total occurrence & $3(6.00)$ & $8(16.00)$ & 5.053 & 0.037 \\
\hline
\end{tabular}

Comparison of complications between two groups; the incidence of complications in PFNA group was significantly lower than that in FHR group and the difference was statistically significant $(p<0.05$, Table 4).

IFF is usually a lower limb fracture caused by direct violence or indirect violence and the incidence rate of women is significantly higher than that of $\operatorname{men}^{[11]}$. Relevant studies showed that after IFF occurs in the body, if active and effective treatment was not given, the patient was likely to die, while if the patient received surgical treatment within $48 \mathrm{~h}$ after fracture, the clinical efficacy can be significantly improved ${ }^{[12]}$. At present, surgical treatment of IFF has reached a clinical consensus, which not only effectively shorten the bed rest time of patients and restore their mobility, but also reduce the incidence of complications ${ }^{[13]}$. PFNA and FHR are two commonly used surgical methods in clinical application at present and their advantages are different ${ }^{[14,15]}$. However, there are few studies on comparing the clinical efficacy of PFNA and FHR on IFF and their effects on TRACP-5b and BALP in China.
Li Zhongtan showed that the weight bearing time and hospitalization time of FHR treatment were significantly shorter than PFNA, but the operation time and intraoperative blood loss were significantly longer than PFNA. In this study, compared with PFNA group, the operation time and intraoperative blood loss in FHR group increased, while the postoperative weight bearing time and hospitalization time were shortened, which was completely consistent with the results of Li Zhongtan's study ${ }^{[16]}$. PFNA, as a new type of internal fixation, is not only simple to operate and PFNA reduces the damage to periosteum in the closed reduction of proximal femur, which is conducive to the preservation of blood supply and further reduces the amount of blood loss during operation ${ }^{[17]}$. Bone cement in FHR can quickly obtain mechanical stability, so patients does not need to wait until fracture healing before bearing weight and they can get out of bed and move as soon as possible, which also plays a positive role in shortening hospitalization time ${ }^{[18]}$. However, the femoral head in FHR operation takes a certain time to wait for bone cement to play its role, reaming, fixation, reduction, etc., so its operation time is relatively long. 
However, with the improvement of the operator's technology and the increase of assistant cooperation, its operation time will be significantly shortened.

Harris rating scale is an authoritative scale for clinical evaluation of hip joint function. The higher the score, the better the recovery effect of hip joint function ${ }^{[19]}$. Ma Gang ${ }^{[20]}$ showed that after FHR surgery, Harris score of patients is significantly higher than PFNA. In this study, Harris scores of both groups increased after operation, but the increase of FHR was significantly higher than that of PFNA group, which was basically consistent with the study of Ma Gang, indicating that both PFNA and FHR can effectively improve hip joint function of IFF patients and the improvement of FHR is more significant. PFNA mostly uses small incision, often does not perform reduction during operation and does not fix the large and small trochanter and lateral wall, thus having certain influence on the recovery of hip joint ${ }^{[21]}$. FHR surgery can effectively restore the mechanical stop point of gluteus medius muscle and iliopsoas muscle by binding and fixing the lateral wall, minor trochanter and major trochanter with steel wire, which plays an important role in maintaining the strength of gluteus medius muscle and iliopsoas muscle, thus effectively improving the hip joint function of patients $^{[22]}$.

Abnormal bone metabolism is one of the main causes of IFF. TRACP-5b and BALP are common clinical bone metabolism indexes and TRACP-5b can reflect osteoclast activity. BALP, as a bone formation specific enzyme in the body, can reflect the osteogenic activity of osteoblasts $^{[23]}$. The research results of Gong Yongshun et $a .^{[24]}$ show that after effective treatment, the level of TRACP-5b in IFF patients decreases and the level of BLAP increases. In this study, the level of TRACP$5 \mathrm{~b}$ decreased and the level of BLAP increased in the two groups after surgery, while the change range in the FHR group was more significant, which was basically consistent with that of Gong Yongshun. Both PFNA and FHR can effectively improve bone metabolism indicators in IFF patients and the improvement effect of FHR is more obvious. PFNA belongs to intramedullary fixation, which has obvious biomechanical advantages. In addition, PFNA realizes antirotation stable support through spiral blades, which can effectively reduce the loss of bone mass in the body. FHR uses artificial femoral head to replace the original femoral head, which can effectively restore the hip joint function of the body, increase the activity of osteoblasts and destroy the activity of osteoclasts, thus effectively improving the bone quality of the body and strengthening bone metabolism.

Postoperative complications are one of the important reasons leading to poor prognosis of patients. Qiao Xiaolu et al. ${ }^{[25]}$ showed that the incidence of complications in patients undergoing PFNA surgery is significantly lower than FHR. In this study, compared with FHR group, the incidence rate of PFNA complications is significantly lower, which is completely consistent with the study of Qiao Xiaolu, indicating that PFNA has more significant advantages in reducing and occurring risks than FHR. FHR is traumatic, difficult to operate and has higher requirements for the operator, which can lead to intolerance of patients and easily lead to complications such as thrombosis and pulmonary infection. PFNA is easy to operate, which has good antirotation and anti-cutting effects and it plays an important role in maintaining the stability and healing of fractures.

To sum up, both PFNA and FHR can effectively treat IFF and improve the levels of TRACP-5b and BALP. PFNA can effectively reduce the operation time, intraoperative blood loss and complication rate. FHR has short postoperative weight bearing time and hospitalization time, which is more conducive to the recovery of hip joint function and better improvement effect on bone metabolism indexes. The two surgical methods have different advantages and can be selected clinically according to the specific conditions of patients.

\section{Conflicts of interest:}

The authors report no conflicts of interest.

\section{REFERENCES}

1. Kandel PR, Pathak L, Singh GP, Baral R. Minimal invasive dynamic hip screw fixation for intertrochanteric fractures of femur. J Univ Coll Med Sci 2016;4(1):26-31.

2. Karakus O, Ozdemir G, Karaca S, Cetin M, Saygi B. The relationship between the type of unstable intertrochanteric femur fracture and mobility in the elderly. J Orthop Surg Res 2018;13(1):207-9.

3. Chang SM, Hou ZY, Hu SJ, Du SC. Intertrochanteric femur fracture treatment in Asia: what we know and what the world can learn. Orthop Clin North Am 2020;51(2):189-205.

4. Dong HP, Huang WJ, Hua J, Dai MH, Xue JW, Yang ZL, et al. Clinical efficacy of artificial femoral head replacement in treating unstable intertrochanteric fracture of femur in advanced aged. Guangxi Med J 2019;41(2):165-9.

5. Baek SH, Baek S, Won H, Yoon JW, Jung CH, Kim SY. Does proximal femoral nail antirotation achieve better outcome than previous-generation proximal femoral nail. World J Orthop 2020;11(11):483-91.

6. Qian J, Wang D, Mei X, Chen J. Therapeutic effect of artificial femoral head replacement and proximal femoral nail 
antirotation on elderly unstable intertrochanteric fractures. Int J Clin Med 2020;11(4):135-43.

7. Li PX, Liu XF, Jiang L, Sun S, Liang Z, Yang L, et al. Effect of Taohong Siwu decoction on fracture healing of osteoporotic intertrochanteric fracture after PFNA. Shandong Med J 2018;58(16):67-9.

8. Huo ZY, Wu F, Liu XW. Observation on the curative effect of Guyifang on intertrochanteric fracture of femur after operation. Chin J Gerontol 2020;40(4):797-800.

9. Wang Y, Pan J. Standard bone cement reinforced PFNA treatment of unstable intertrochanteric fractures in the elderly. Chin J Orthop Surg 2018;26(18):1653-8.

10. Theil C, Roedl R, Gosheger G, Moellenbeck B, Frommer A, Dieckmann R, et al. Total joint replacement of the hip and knee in patients with arthrogryposis multiplex congenita: a report of six joints. Arch Orthop Trauma Surg 2020:1-8.

11. Jha V, Ahmed T. Modified short proximal femoral nail for intertrochanteric fractures of femur in indian patients-our experience. Malays Orthop J 2020;14(2):72-82.

12. Werner BC, Fashandi AH, Gwathmey FW, Yarboro SR. Trends in the management of intertrochanteric femur fractures in the United States 2005-2011. Hip Int 2015;25(3):270-6.

13. Chen J, Zheng Y, Xing Y, Lin Y, Zhong W. Comparative analysis of three internal fixation methods for intertrochanteric fracture of femur in the elder. Int J Clin Exp Med Sci 2020;6(1):5-11.

14. Chang HM, Lu WY, Kuan FC, Su WR, Chen PY, Su PF, et al. Wound drainage after proximal femoral nail antirotation (PFNA) fixation may negatively affect the patients with intertrochanteric fractures: A prospective randomized controlled trial. Injury 2021;52(3):575-81.

15. Zhou Y, Tang M, Xu KY. Clinical analysis of PFNA and artificial femoral head replacement in the treatment of intertrochanteric fracture in the elderly. Chin J Bone Joint Inj 2019;34(4):376-8.

16. Li ZT. Comparative analysis of the curative effect of artificial femoral head replacement and PFNA in the treatment of unstable intertrochanteric fracture of the elderly. Hebei Med J 2018;24(2):188-92.

17. Kammerlander C, Hem ES, Klopfer T, Gebhard F, Sermon A, Dietrich M, et al. Cement augmentation of the Proximal Femoral Nail Antirotation (PFNA)-A multicentre randomized controlled trial. Injury 2018;49(8):1436-44.
18. Wang SF, Ji QH, Qiao XF, Zhao P, Xue Y, Li YB. Efficacy of artificial femoral head replacement for femoral head avascular necrosis. Medicine 2019;98(17):e15411-13.

19. Buker N, Eraslan U, Kitis A, Kiter AE, Akkaya S, Sutcu G. Is quality of life related to risk of falling, fear of falling and functional status in patients with hip arthroplasty. Physiother Res Int 2019;24(3):e1772.

20. Ma G. Comparison of short term efficacy of two methods in the treatment of intertrochanteric fracture of femur in the elderly. Biol Orthop Mater Clin Res 2018;15(6):55-7.

21. Zhou S, Liu J, Zhen P, Shen W, Chang Y, Zhang H, et al. Proximal femoral nail anti-rotation versus cementless bipolar hemiarthroplasty for unstable femoral intertrochanteric fracture in the elderly: a retrospective study. BMC Musculoskelet Disord 2019;20(1):500-2.

22. Zhong CL, Zhou W, Zhou F, Zhu SQ, Wang Y. Clinical effect of PFNA and FHR in the treatment of senile patients with unstable intertrochanteric fractures. J Trauma Surg 2018;20(9):686-9.

23. Terpos E, Fotiou D, Anargyrou K, Vassilakopoulos TP, Christoulas D, Makras P, et al. Bone loss and high bone turnover in patients with Non-Hodgkin's lymphoma who receive frontline chemotherapy: Final results of a multicenter prospective study. Blood 2019;134(1):4124-6.

24. Gong YS, Wan $\mathrm{C}, \mathrm{Xu}$ Y. Clinical efficacy of parathyroid hormone combined with low frequency pulsed electromagnetic field on osteoporotic fractures of the femur in elderly patients. Chin J Osteoporos 2018;24(1):1-4.

25. Qiao XL, Dai QQ. Comparison of FHR and PFNA in the treatment of intertrochanteric fractures in the elderly. J Lab Med Clin Sci 2019;16(14):2075-7.

This is an open access article distributed under the terms of the Creative Commons Attribution-NonCommercial-ShareAlike 3.0 License, which allows others to remix, tweak, and build upon the work non-commercially, as long as the author is credited and the new creations are licensed under the identical terms

This article was originally published in a special issue, "Evolutionary Strategies in Biomedical Research and Pharmaceutical Sciences" Indian J Pharm Sci 2021:83(3) Spl issue;127-132 